\title{
Numerical Simulation on the Heat Dissipation of the Outdoor Unit in the Groove of High-Rise Building
}

\author{
Yuan Wang ${ }^{1,2^{*}}$, Xiaoqing Zhou ${ }^{2}$, Ju Tian ${ }^{1}$, Chuansheng Liu ${ }^{1}$ and Jian Wang ${ }^{1}$ \\ ${ }^{1}$ School of Aircraft Maintenance Engineering, Guangzhou Civil Aviation College, Guangzhou, 510403, China \\ ${ }^{2}$ School of Civil Engineering, Guangzhou University, Guangzhou, 510006, China
}

\begin{abstract}
In order to study the influence of the heat dissipation of the outdoor unit in the groove of the highrise building. Numerical simulation of the thermal environment of a high-rise building is carried out. The results show that: When the outdoor mean wind speed is $2.7 \mathrm{~m} / \mathrm{s}$, the maximum inlet air temperature of the outdoor unit under full load condition is about $43.3^{\circ} \mathrm{C}$, which is $2{ }^{\circ} \mathrm{C}$ lower than that under no wind condition. The monsoon is beneficial to the heat dissipation of the outdoor unit. When the hot air in the building groove rises, the corner flow is induced at the lower side of the refuge layer. Due to the reverse pressure gradient, the flow separation occurs, resulting in the blockage in the flow channel. When the air flows through the refuge layer, the flow expands suddenly due to the increase of the cross-section of the channel. The turbulent boundary layer is separated. The vortex region is induced on the upper side of the refuge layer. Since the heat on the upper and lower sides of the refuge floor cannot be effectively dissipated, the inlet air temperature of the outdoor unit is higher than that of other floors, which reduces the working efficiency of the outdoor unit.
\end{abstract}

\section{Introduction}

Multi-split air conditioning system is highly automated and flexible, which is widely used in high-rise buildings ${ }^{[1-2]}$. Generally, the wind environment directly affects the microclimate of the urban environment and people's comfort, so the wind environment should be considered in the planning and design of super high-rise buildings. However, the structure and distribution of high-rise buildings are relatively complicated. At present, there are few studies on the air distribution of air conditioning systems in super high-rise residential buildings ${ }^{[3-4]}$. Moreover, under the effect of regional monsoon and the interaction between buildings, the study of airflow distribution in the special position of the external structure of the building becomes very complex. Due to the influence of the building groove and the location of the outdoor unit, the ventilation of the outdoor unit is poor. The heat cannot be dissipated in time, which increases the return air temperature of the outdoor unit. In serious cases, the air conditioner shuts down ${ }^{[5-6]}$. Besides, the lateral exhaust or upper exhaust of the outdoor units on low floors may affect the return air temperature environment of adjacent upper units, resulting in an airflow short circuit. Therefore, it is necessary to study the heat dissipation of the outdoor unit in high-rise buildings.

In this paper, PHOENICS numerical simulation software is used to study the distribution of heat dissipation and the working conditions of the outdoor units in the groove of high-rise buildings.

\section{Numerical simulation model}

\subsection{Geometric model}

The building height in the numerical simulation of the thermal environment is $183.15 \mathrm{~m}$. The geometric model of the building is established according to the architectural design plan, and the size and location are consistent with the design plan. The geometric model of the building is shown in Figure 1. The first floor of the building is overhead. The 16 th, 32 nd and 48 th floors are refuge floors, and there are no outdoor units on these three floors; the other floors are equipped with outdoor units. The location of the outdoor unit is shown in Figure 2.

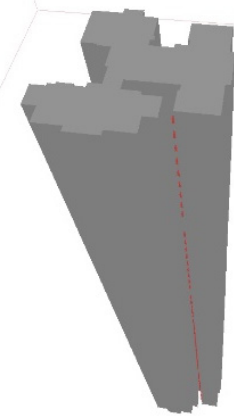

Fig. 1 The building model

\footnotetext{
*Corresponding author: wangyuan_nuaa@163.com
} 


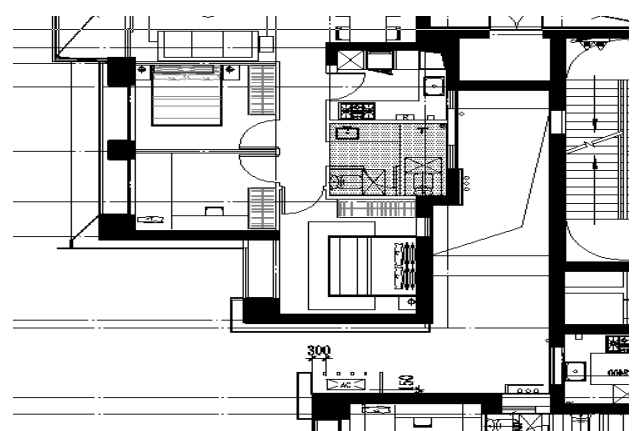

Fig. 2 Location of the outdoor unit

\subsection{Numerical method}

\subsubsection{Turbulence model}

The standard $k-\varepsilon$ turbulence model is selected to simulate the outdoor wind environment of buildings. The second-order upwind scheme is used to discretize the equations. The standard wall function method is used near the wall. The simple algorithm is used for velocity and pressure coupling. The standard $k-\varepsilon$ governing equation is as follows:

$$
\begin{array}{r}
\frac{\partial}{\partial t}(\rho k)+\frac{\partial}{\partial x_{i}}\left(\rho k u_{i}\right)=\frac{\partial}{\partial x_{j}}\left[\left(\mu+\frac{\mu_{i}}{\sigma_{k}}\right) \frac{\partial k}{\partial x_{j}}\right]+ \\
G_{k}+G_{b}-\rho \varepsilon-Y_{M}+S_{k} \\
\frac{\partial}{\partial t}(\rho \varepsilon)+\frac{\partial}{\partial x_{i}}\left(\rho \varepsilon u_{i}\right)=\frac{\partial}{\partial x_{j}}\left[\left(\mu+\frac{\mu_{i}}{\sigma_{k}}\right) \frac{\partial \varepsilon}{\partial x_{j}}\right]+ \\
C_{1 \varepsilon} \frac{\varepsilon}{k}\left(G_{k}+G_{3 \varepsilon} G_{b}\right)-C_{2 \varepsilon} \rho \frac{\varepsilon^{2}}{k}+S_{\varepsilon}
\end{array}
$$

\subsubsection{Distribution of inlet wind speed}

In order to make the simulation close to the actual situation, the outdoor wind speed varies with the height. The wind speed at the entrance of the near surface layer obeys the exponential distribution.

$$
u_{h}=u_{0}\left(\frac{h}{h_{0}}\right)^{a}
$$

Where:

$u_{h} \quad$ The wind speed at $h$ height;

$u_{0} \quad$ The wind speed at $h_{0}$ height;

$h_{0}$ The reference height of meteorological typhoon observation point. Generally, the value is $10 \mathrm{~m}$;

$a$ A constant related to the type of ground roughness. The value of $a$ in this paper is 0.2 .

According to the meteorological parameters of Shenzhen, the dominant wind direction in summer is a southeast wind, and the outdoor mean wind speed is $2.7 \mathrm{~m} / \mathrm{s}$. According to formula (2), the gradient wind boundary conditions are set.

In this paper, two cases are simulated: 1 . there is no wind in the environment; 2 . the outdoor mean wind speed is $2.7 \mathrm{~m} / \mathrm{s}$.

\subsubsection{Boundary conditions}

According to the design code for heating ventilation and air conditioning of civil buildings, the outdoor temperature in summer in Shenzhen can be used as the simulation temperature, which is $33.7^{\circ} \mathrm{C}$. In order to simplify the model, the following assumption is adopted: the building envelope can be regarded as an adiabatic boundary. Solar radiation, ground reflection and heat transfer between the walls are ignored. The outdoor unit of the air conditioner runs at full load.

The relevant parameters of the outdoor unit are shown in Table 1.

Table 1. The parameters of the outdoor unit

\begin{tabular}{|c|c|c|c|c|}
\hline $\begin{array}{c}\text { Heat dissipation } \\
(\mathrm{KW})\end{array}$ & $\begin{array}{c}\text { Exhaust } \\
\text { velocity } \\
(\mathrm{m} / \mathrm{s})\end{array}$ & $\begin{array}{c}\text { Exhaust air } \\
\text { rate } \\
\left(\mathrm{m}^{3} / \mathrm{min}\right)\end{array}$ & $\begin{array}{c}\text { Size } \\
(\mathrm{mm})\end{array}$ & $\begin{array}{c}\text { Outlet } \\
\text { diameter } \\
(\mathrm{mm})\end{array}$ \\
\hline 22.063 & 4.1 & 2.04 & $1380 \times 950 \times 370$ & 560 \\
& & & & \\
\hline
\end{tabular}

\section{Results and discussion}

\subsection{Heat dissipation distribution in the building groove}

Numerical simulation analysis is carried out for the highrise building under wind $(2.7 \mathrm{~m} / \mathrm{s})$ and no wind conditions. The temperature distribution at different building heights is shown in the following figure:

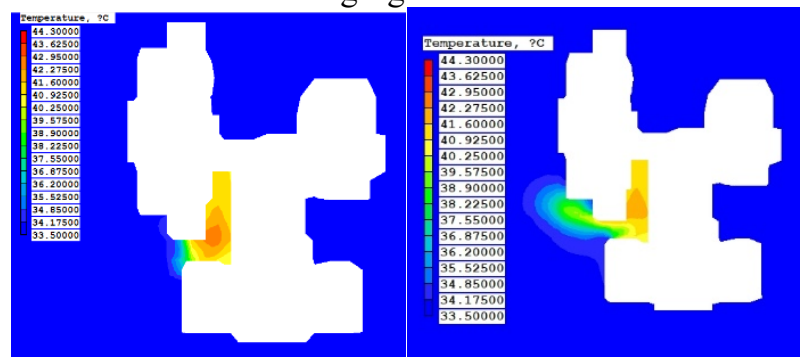

(1) Mean wind speed $(2.7 \mathrm{~m} / \mathrm{s})$

(2) No wind

Fig. 3 Temperature distribution at a height of $170 \mathrm{~m}$

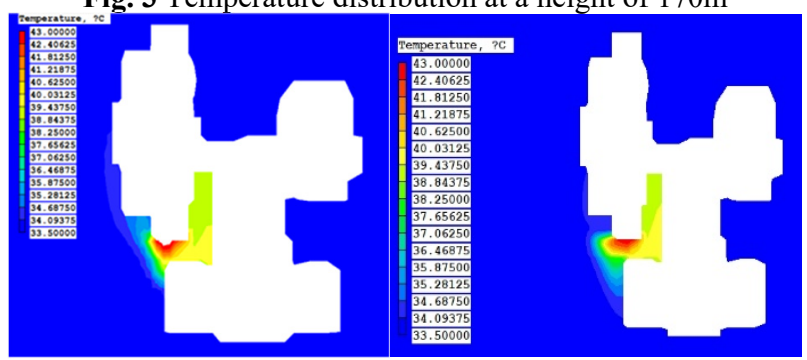

(1) Mean wind speed $(2.7 \mathrm{~m} / \mathrm{s})$

(2) No wind

Fig. 4 Temperature distribution at a height of $100 \mathrm{~m}$ 


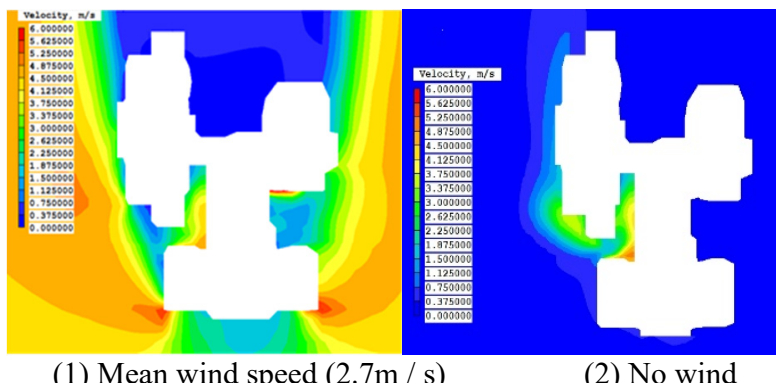

(1) Mean wind speed $(2.7 \mathrm{~m} / \mathrm{s})$

(2) No wind

Fig. 5 Velocity distribution at a height of $170 \mathrm{~m}$

It can be seen from the above three figures that the outdoor unit in the groove is placed on one side, and the temperature distribution is uneven. In general, the temperature in the area near the outdoor unit is higher.

In windy conditions, the maximum temperature can reach $44.2^{\circ} \mathrm{C}$, and the maximum temperature appears on the wall facing the direction of the outdoor unit exhaust. This is because the wall is only $1.8 \mathrm{~m}$ away from the exhaust louvers of the outdoor unit, and the exhaust air of the outdoor unit blows directly on the wall, causing heat to accumulate near the wall, making the temperature higher than the ambient air temperature. The temperature at other locations in the groove is slightly lower than that of the exhaust area of the outdoor unit, but higher than the outdoor environment temperature. This is because the fluidity in the groove is relatively poor compared to the outside of the building. Due to poor heat dissipation, heat accumulates near the installation location of the outdoor unit, and the intake and exhaust temperatures are relatively high. The airflow outside the building groove is influenced by the monsoon and other building structures, so the heat can't spread out smoothly. There is a low velocity region. Under the condition of no wind, the maximum temperature in the groove can reach $46^{\circ} \mathrm{C}$. Due to the high exhaust speed of the outdoor unit, the heat diffuses to both sides, and the influence range of high temperature area is larger than that under the windy condition.

\subsection{Analysis of the working condition of the outdoor unit in the building groove}

As shown in Fig. 6, the temperature distribution in the groove is as follows: the temperature at the bottom of the groove is relatively low, and the temperature distribution increases with the increase of the height, regardless of the working conditions with or without wind. When the airconditioning equipment is running, the heat of the outdoor unit is directly discharged into the air. Due to the effect of thermal buoyancy, the ambient temperature of the upper layer of the building rises, which affects the operational performance of the air conditioner on the upper floor. The maximum temperature in the groove under no wind condition is slightly $2^{\circ} \mathrm{C}$ higher than that under wind condition, and the influence range of high temperature area is larger. The reason is that under the influence of monsoon, the velocity of airflow in the groove is accelerated. The heat accumulated in the groove is dispersed to the external environment, which makes the temperature in the groove drop.

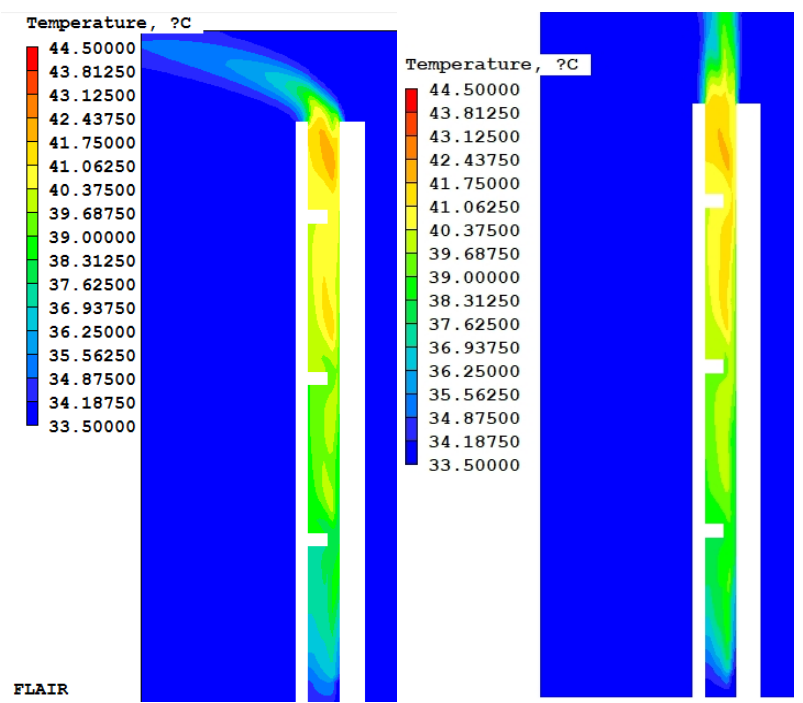

(1) Mean wind speed $(2.7 \mathrm{~m} / \mathrm{s})$

(2) No winc

Fig.6 Temperature distribution at the middle section of building groove

Figure 7 - (1) shows the temperature distribution at the air inlet on the right side of the outdoor unit under windy condition. It can be observed in the figure that the upper and lower sides of the 48th floor of the refuge layer are affected by high temperature airflow, and the high temperature area covers 44-52 floors. Among them, the temperature at the side air inlet of the outdoor unit on the 50th and 51st floors is higher, and the maximum temperature is about $43.3^{\circ} \mathrm{C}$. The inlet air temperature of the outdoor units on the other floors is close to $43{ }^{\circ} \mathrm{C}$. The influence range of the high temperature area near the $32 \mathrm{nd}$ floor of the refuge floor is 28-35 floors; Among them, the temperature at the side air inlet of the outdoor unit on the 33rd and 34th floors is relatively high, with the highest temperature being about $43.2^{\circ} \mathrm{C}$, and the inlet air temperature of the outdoor unit on the other floors is close to $42^{\circ} \mathrm{C}$. In general, the inlet air temperature of each outdoor unit increases with the increase of height. For the units near the upper and lower sides of the refuge floor, the inlet air temperature will be slightly higher than that of the nearby outdoor unit due to the influence of airflow around the refuge layer. It can be seen from Fig. 7 - (2) that the temperature distribution at the inlet of the right side of the outdoor unit under no wind condition is similar to that under wind condition. However, the inlet temperature of some outdoor units has reached $45.3^{\circ} \mathrm{C}$. Due to the high condensation temperature of the air conditioner, shutdown protection may occur, which seriously affects the normal operation of the outdoor unit. 


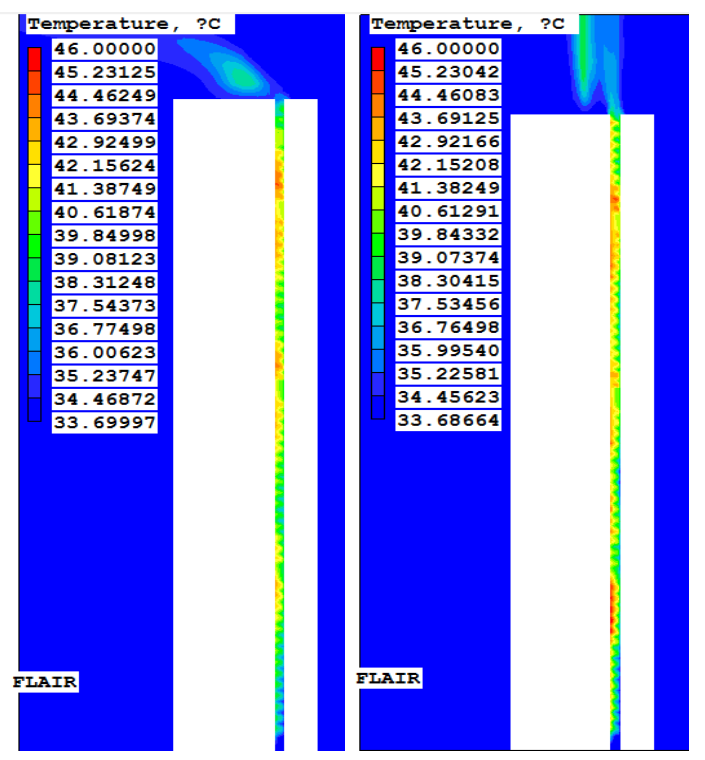

(1) Mean wind speed $(2.7 \mathrm{~m} / \mathrm{s})$

(2) No wind

Fig.7 Temperature distribution at the side air inlet of the outdoor unit in the groove

As shown in Fig. 8 and Fig. 9, when the hot air in the building groove rises, the corner flow is induced at the lower side of the refuge layer. Due to the reverse pressure gradient, the flow separation occurs, resulting in the blockage of the flow channel. When the air flows through the refuge layer, the flow expands suddenly due to the increase of the cross-section of the channel. The turbulent boundary layer is separated. The vortex region is induced on the upper side of the refuge layer. Since the heat on the upper and lower sides of the refuge floor cannot be effectively dissipated, the inlet air temperature of the outdoor unit is higher than that of other floors, which reduces the working efficiency of the outdoor unit.

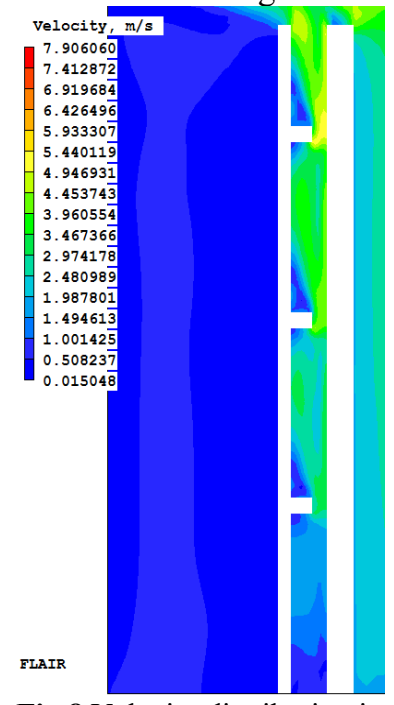

Fig.8 Velocity distribution in the groove

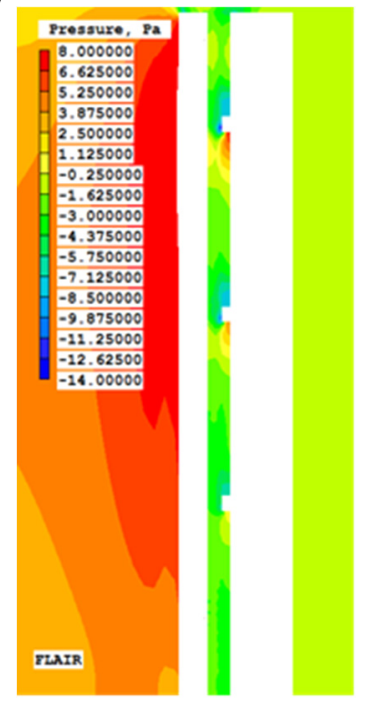

Fig.9 Pressure distribution in the groove

\section{Conclusion}

In this paper, the numerical investigation of the thermal environment of the outdoor units in the groove of high-rise building is carried out. It is found that it is affected by the outdoor wind speed and the shape of the building structure.
The conclusions are as following:

1. When the outdoor mean wind speed is $2.7 \mathrm{~m} / \mathrm{s}$, the maximum inlet air temperature of the outdoor unit under full load condition is about $43.3{ }^{\circ} \mathrm{C}$, which is $2{ }^{\circ} \mathrm{C}$ lower than that under no wind condition. The monsoon is beneficial to the heat dissipation of the outdoor unit.

2. When the hot air in the building groove rises, the corner flow is induced at the lower side of the refuge layer. Due to the reverse pressure gradient, the flow separation occurs, resulting in the blockage of the flow channel. When the air flows through the refuge layer, the flow expands suddenly due to the increase of the crosssection of the channel. The turbulent boundary layer is separated. The vortex region is induced on the upper side of the refuge layer. Since the heat on the upper and lower sides of the refuge floor cannot be effectively dissipated, the inlet air temperature of the outdoor unit is higher than that of other floors, which reduces the working efficiency of the outdoor unit.

\section{References}

1. Zhou Dehai, Shao Xiaoliang, Zhang Xiaoling, et al. Numerical simulation of thermal environment for outdoor unints at building reentrant $[\mathrm{J}]$. Journal of Guangzhou University(Natural Science Edition), 2010, 9(6) :1722.

2. Jiang Yuebo, Jin Wufeng, Piao Wankui, et al . CFD analysis and optimization of thermal environment for outdoor units at re-entrant of residential building $[\mathrm{J}]$. Building Energy Conservation, 2012, 40( 9) :4-8.

3. Jin Wufeng, Zheng Yafei, Jiang Yuebo, Bai Xiansan. Experimental study on the effect of the outdoor unit installation conditions on the heat dissipation in the building groove $[\mathrm{J}]$. Journal of Xi'an University of Architecture \& Technology(Natural Science Edition), 2015, 47(2) :282-286.

4. Hang Yin, Liu Dong, Huang Yan, Liu Jun. Numerical Simulation of Airflow and Optimization of Outdoor Unit of the Air Conditioner in a Certain High Building [J]. Building Energy \& Environment, 2006, 25(3) :12-16.

5. Shen Lin, Wan Xiongfeng. Simulation of the Operating Environment around Outdoor Air Conditioner Unit in a High Rise Building [J]. Building Energy \& Environment, 2010, 29(4) :8288.

6. Jia Xuefeng, Liu Dong. Numerical Simulation of Ventilation Effect of Outdoor Units in Air Conditioning System for High-rise Building [J]. Building Energy \& Environment, 2010, 29(1) :69-76. 